\title{
Mesure de la variabilité de la couleur du bois. Nouvelle méthode appliquée aux carottes de sondage.
}

\author{
G. JANIN, J.F. MAZET \\ avec la collaboration technique de C. HOUSSEMENT et A. PERRIN \\ INRA, Station de Recherches sur la Qualité des Bois, Centre de Recherches de NANCY \\ Champenoux, F 54280 Seichamps
}

\begin{abstract}
Résumé
L'importance de la mesure de la couleur du bois d'un arbre sur pied, abattu ou stocké sur le parc à bois, pour l'évaluation de sa qualité et de son prix, nous a amenés à développer la mesure de la couleur après prélèvement d'une carotte de sondage de 5 ou $10 \mathrm{~mm}$ de diamètre.

La carotte de sondage, incluse dans un bloc de bois de hêtre, est ensuite tranchée en feuilles de placage mince $\left(6 / 10^{\mathrm{C}}\right.$ de $\left.\mathrm{mm}\right)$ dans les conditions industrielles. Ces feuilles de placage, qui ont un état de surface comparable à celui des placages industriels obtenus à partir des mêmes billes de bois, servent à la mesure de la couleur à l'aide d'un spectrophotocolorimètre.

Ces mesures permettront de connaître objectivement la couleur des arbres et de procéder à des comparaisons et des classements entre eux, dans les différents systèmes de mesure des écarts de couleur CIELAB ou HUNTER L, a, b.
\end{abstract}

Mots clés : Bois, couleur, placages, variabilité, spectrophotométrie, carottes de sondage.

\section{Introduction}

La couleur du bois est un des aspects importants de l'apparence d'un bois massif ou de placage. Elle s'ajoute aux détails de structure du plan ligneux d'une espèce qui apparaissent à la surface d'un placage au cours de l'opération de tranchage et qui permettent souvent de la reconnaître.

L'appréciation de la couleur du bois est donc liée à toutes les impressions visuelles ressenties à l'examen d'un échantillon de placage et devient de ce fait très subjective. Pour pallier à cette source de variation de la perception de la couleur entre différents individus, il faut procéder à des mesures objectives par une quantification de la couleur.

Le bois, par son aspect, sa clarté plus ou moins grande et sa teinte, apporte une sensation colorée appréciée dans la fabrication des meubles, des lambris ou parquets et des panneaux de décoration. 
Cet aspect existe aussi bien avec des feuilles minces de placages de bois obtenues par le tranchage des grumes que lors de l'utilisation de bois massif.

Le tranchage est l'opération technologique de transformation du bois qui valorise le plus le bois en le divisant en feuilles minces qui multiplient sa surface visible et permetttent de produire des panneaux de placages pour meubles de haute qualité.

Lorsqu'un placage présente un bel aspect, soit un droit fil provenant du débit sur dosse avec des dessins ou arcatures donnés par la coupe longitudinale tangentielle des accroissements annuels, soit une figuration sur mailles due à la présence des rayons ligneux, nous savons que les industriels lui donnent une valeur d'autant plus grande qu'il possède, comme dans le cas du chêne, une grande clarté ou luminosité et une teinte jaune paille claire.

En effet, le prix au $\mathrm{m}^{3}$ de chaque grume dépend, d'une part de critères de qualité externes qui déterminent le choix de l'acheteur : longueur, diamètre, rectitude, absence de défauts comme les nœuds superficiels, profonds, ou les fentes..., et d'autre part des caractéristiques internes qui sont difficiles, voire impossibles à apprécier d'une manière directe autant pour un arbre sur pied que sur la grume abattue : le grain conditionné par la largeur des cernes, leur régularité, et surtout l'homogénéité de la couleur. Celleci doit être claire (luminance $\mathrm{L}^{*}$ élevée) et régulière tout au long de la grume.

Le prix $\mathrm{du} \mathrm{m}^{3}$ de la grume ou du placage obtenu peut varier de 1 à 5 fois suivant son aspect final, sa clarté et sa couleur pour la fourniture des placages dans les meubles.

Jusqu'à ce jour, l'appréciation de la couleur d'une grume de tranchage abattue en forêt était faite sur la section ou sur une découpe fraîche en l'humidifiant à l'eau, ou encore en pratiquant une entaille oblique avec une hachette pour enlever l'écorce, le bois d'aubier et mettre à jour le bois de cœur.

Ces méthodes appliquées sur le terrain ne permettent pas de bien juger ni de la couleur du bois massif en grume, et à plus forte raison de la couleur finale du placage, ni de comparer des grumes de tranchage entre elles.

Nous proposons de mesurer objectivement la couleur du bois des arbres sur pied, des grumes abattues en forêt ou stockées sur les parcs à bois en usine, en utilisant les carottes de sondage prélevées à la tarière de Pressler, qui sont des prélèvements non destructifs de $10 \mathrm{~mm}$ ou de $5 \mathrm{~mm}$ de diamètre dans l'arbre.

De plus, pour obtenir une mesure de la couleur du bois qui tienne compte d'un état de surface comparable au placage final lors du tranchage industriel, nous décrivons ci-dessous une méthode de tranchage des carottes de sondage dans les mêmes conditions qu'en usine et qui conduit à un état de surface et à une "figuration " identiques à ceux des placages éventuels issus de la même grume.

Cette mesure de la couleur du bois sur carottes de sondage fait suite aux méthodes de mesures non destructives développées depuis longtemps à la Station de Recherches sur la Qualité des Bois sur les mêmes types d'écharitillons (Thiercelin et Perrin, 1972 ; Janin, 1973 ; Polge, 1978 ; Polge et Thiercelin, 1979 ; Bucur, 1983 ; Janin, 1983 ; Perrin, 1983 ; Gentner, 1985).

La facilité de prélèvement des carottes de sondage, leur grand nombre et la commodité de mise en cuvre en font un outil adapté aux études de populations dans des plans d'expérience sur le terrain pour les études forestières : comparaisons d'espèces, de provenances ou de tests de descendances. 


\section{Matériel et méthodes de tranchage des carottes de sondage}

Dès que la carotte de sondage est prélevée, elle est mise dans une chambre conditionnée en température $20^{\circ} \pm 1^{\circ}$ et à une humidité relative de $65 \% \pm 5 \%$. Il faut ensuite la présenter dans un montage d'usinage pour être tranchée sur une machine industrielle.

\subsection{Montage d'usinage}

Les carottes de sondage sont placées dans des boîtiers en bois de hêtre qui présente la particularité de se trancher très régulièrement et de bien se prêter à l'usinage.

Les boîtiers de hêtre sont composés de deux planches rainurées à la toupie dans le sens du fil du bois à l'aide de deux fers profilés pour les carottes de $10 \mathrm{~mm}$ et celles de $5 \mathrm{~mm}$ de diamètre.

Les carottes sont alors enduites de colle et disposées dans les rainures d'une planche, et l'autre planche est rabattue, collée à la colle vinylique et pressée pour le séchage afin de donner un bloc compact contenant les carottes positionnées pour le sens du tranchage choisi : sur dosse ou sur quartier (voir schéma).

Durant l'usinage du hêtre, les faces de référence ont été soigneusement repérées pour obtenir un parfait vis-à-vis des deux rainures. Un plan du bloc de hêtre donne la numérotation des échantillons.

On a pu ainsi, à titre d'exemple, disposer dans un bloc de $60 \times 30 \times 10 \mathrm{~cm}, 50$ carottes de $10 \mathrm{~mm}$ et 50 carottes de $5 \mathrm{~mm}$.

\subsection{Le tranchage}

Les épaisseurs de placage industriel en usage courant sont de $6 / 10^{\mathrm{c}}$ de $\mathrm{mm}$. Ainsi, le bloc de hêtre dans lequel se trouvent placées les carottes de sondage sera débité en tranches de $6 / 10^{\mathrm{c}}$ de $\mathrm{mm}$ et fournira: diamètre ;

- de 8 à 10 tranches utilisables pour les carottes de sondage de $10 \mathrm{~mm}$ de

- de 4 à 5 tranches utilisables pour les carottes de sondage de $5 \mathrm{~mm}$ de diamètre.

Après le tranchage, les feuilles de placages sont mises sous presses pour être aplanies et servir aux mesures.

\subsection{Appareillage et conditions de mesure}

Nous avons utilisé pour nos mesures un spectrophotocolorimètre du type COLORQUEST de HUNTERLAB qui est couplé à un calculateur I.B.M. PC avec des disquettes programmées, relié à une imprimante (JANIN, 1985, 1986a, 1986b).

Le COLORQUEST fournit les données colorimétriques dans différents systèmes de couleurs (HUNTER, 1975) : 

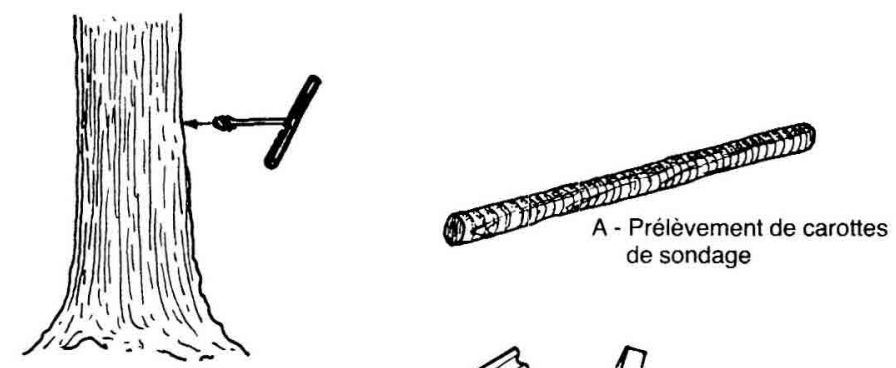

B - Mise en place des carottes dans les rainures
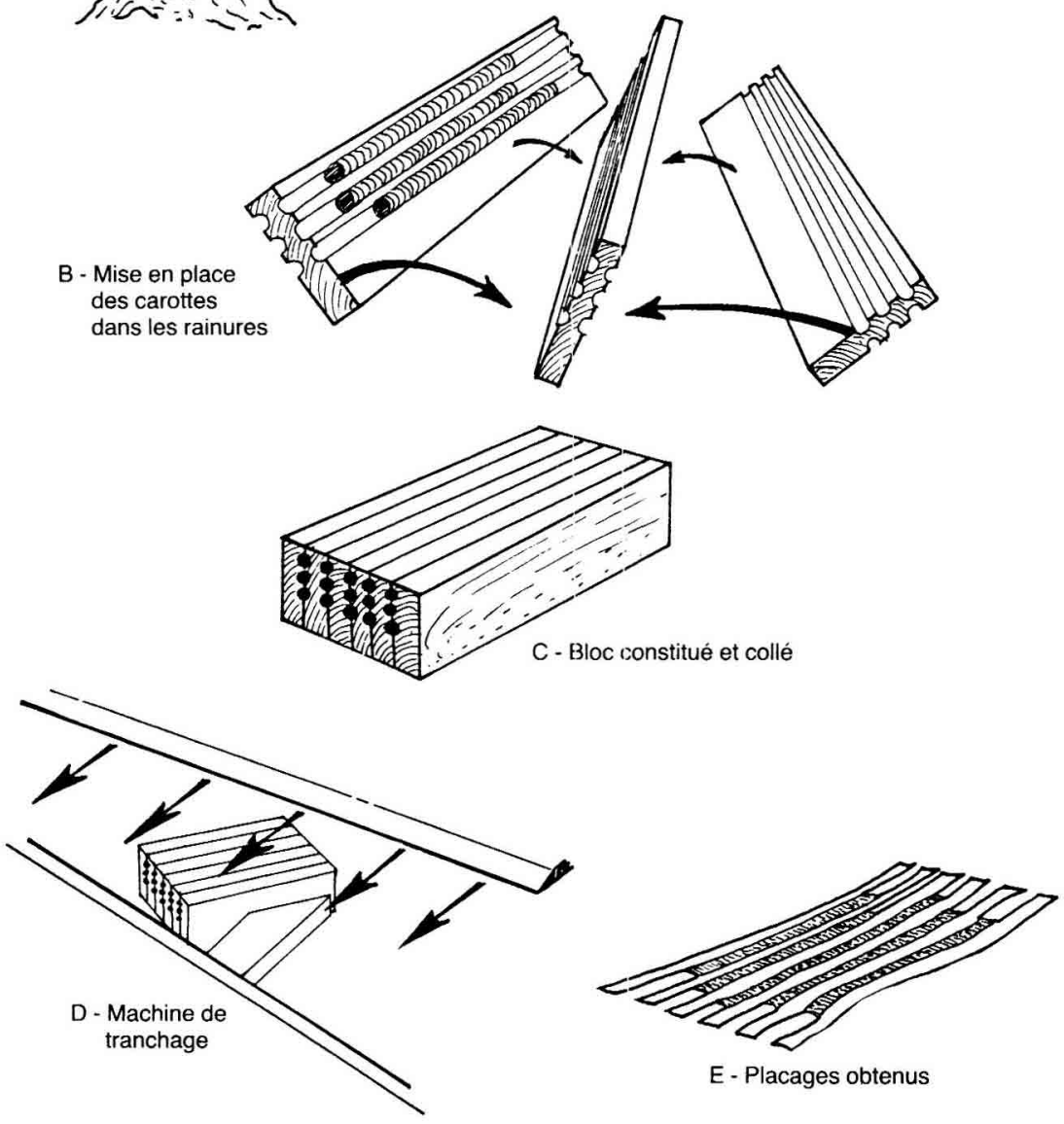

E - Placages obtenus

FIG. 1

Diagram of differents steps of recovering veneers from wood increment cores 10 and $5 \mathrm{~mm}$ thickness.

Schéma des différentes étapes d'obtention de placages à partir de carottes de sondage $(5 \mathrm{~mm}$ ou $10 \mathrm{~mm}$ de diamètre).
A : Increment cores sampling.
B : Ordering cores in wood grooved.
C: Glued wood block.
$\mathrm{D}$ : Veneering machine and knife.
$\mathrm{E}$ : Obtained cores veneers. 
- X, Y, Z : valeurs tristimulaires,

- luminosité, L : $f(\lambda)$,

- $x, y$, coordonnées chromatiques dans le diagramme C.I.E.,

- Iongueur d'onde dominante et pureté de la teinte,

- HUNTER L, a, b,

- $\operatorname{CIELAB}\left(\mathrm{L}^{*}, \mathrm{a}^{*}, \mathrm{~b}^{*}\right)$,

- divers indices: de blancheur, de jaune et de métamérisme, et ceci pour différents illuminants standards de notre choix : A, B, C, $\mathrm{D}_{65} \ldots$

Les fenêtres de mesure du porte-échantillon sont de deux types :

- $25 \mathrm{~mm}$ d'ouverture pour des échantillons larges,

- $9 \mathrm{~mm}$ pour des échantillons de forme étroite.

Le premier type permet d'intégrer une large surface de l'échantillon et de donner une bonne indication de la couleur d'un échantillon assez uniforme.

Le deuxième type est utilisé pour des échantillons qui présentent des variations locales d'aspect ou de couleur sur leur surface. Il nécessite un réducteur optique de visée pour observer avec précision une plage plus intéressante et exige une nouvelle calibration pour cette surface réduite. Nous l'utilisons pour la mesure de carottes de $5 \mathrm{~mm}$ de diamètre en adaptant un diaphragme convenable à la dimension de l'échantillon après tranchage.

On peut ainsi choisir de mesurer la moyenne des coordonnées chromatiques $L$, a, $\mathrm{b}$, tout au long de la carotte, ou un groupe de cernes donné sur la carotte de sondage.

\section{Résultats}

Les espèces les plus utilisées pour le tranchage sont, par ordre d'importance : le chêne, le noyer, le merisier, lorme, ou divers arbres fruitiers. La destination d'une bille de bois vers le tranchage modifie beaucoup son prix et a pour origine l'homogénéité particulière de sa couleur, la qualité des veines colorées (noyer) ou laspect ondé du plan ligneux des fibres (érable, merisier, ... bois tropicaux) nécessaires à l'utilisation en ébénisterie et marqueterie.

Les mesures ont été effectuées sur les feuilies de placages découpées à partir de carottes de sondage de bois de chêne destinés au tranchage.

Dans le cas des carottes de $5 \mathrm{~mm}$ de diamètre, pour éviter l'influence sur la mesure de la couleur du phénomène superficiel d'oxydation par l'air, qui se manifeste aussi par une coloration parasite due au métal (Fe) dont la tarière de Pressler est constituée, nous ne prenons en compte que les feuilles de placages correspondant à la zone du plus grand diamètre, soit 2 ou 4 feuilles de placages.

En revanche, la mesure de la couleur pratiquée sur les carottes de $10 \mathrm{~mm}$ n'est pas affectée par le même phénomène parce que la largeur des placages utilisables est suffisamment grande pour pouvoir s'affranchir des effets de bords. 
Le tableau ci-dessous montre que les différences entre 22 arbres peuvent être mises en évidence à l'aide des caractéristiques chromatiques sous illuminant A aussi bien sur les carottes de $10 \mathrm{~mm}$ et de $5 \mathrm{~mm}$ débitées en placages :

\begin{tabular}{|c|c|c|c|c|}
\hline Carottes $F_{n_{1}}^{n_{1}} \ldots \ldots \ldots \ldots \ldots \ldots$ & Seuil & $\mathrm{L}^{*}$ & $a^{*}$ & $b^{*}$ \\
\hline de $5 \mathrm{~mm} \mathrm{~F} \underset{t h}{21}$ & $1 \%$ & 17,7 & 23,9 & $1,12 \mathrm{NS}$ \\
\hline de $10 \mathrm{~mm} \mathrm{~F} \frac{21}{19 x}$ & $1 \%$ & 131.8 & 160,6 & 2,75 \\
\hline
\end{tabular}

Ces valeurs de $F_{n_{1}}^{n_{2}}$ sont toutes significatives au seuil de $1 \%$ sauf pour le caractère $b^{*}$, qui décrit souvent moins bien la couleur des placages dans le domaine du jaunebleu sous l'illuminant $A$. En effet la courbe spectrale de l'illuminant A favorise les composants chromatiques $a^{*}$ dans le rouge-orangé.

D'autre part l'analyse de la variance entre les carottes de $10 \mathrm{~mm}$ et de $5 \mathrm{~mm}$ pour la luminance $\mathrm{L}^{*}$ montre que les mesures ne sont pas significativement différentes entre elles $F_{36}^{l}=3,10 \mathrm{NS}$ (seuil de $1 \%$ ).

Par ailleurs la luminance $\mathrm{L}^{*}$ est un caractère très discriminant pour le classement des bois de placage, placages clairs ou sombres (Moslemi, 1967).

\section{Conclusion}

La couleur du bois est un facteur clé de la qualité (JANIN, 1986 a et b). La mesure et l'appréciation des différences de teintes intéressent aussi bien les industriels, les utilisateurs que les forestiers (JANIN, 1986c).

Ces derniers, dans le but de fournir une production d'arbres de qualité, doivent connaître les forêts où existent les bois de meilleure couleur pour une espèce donnée et aussi déterminer les individus qui possèdent cette couleur appréciée. De cette façon nous pourrons dans l'avenir espérer avoir une action par la sélection génétique ou une meilleure adaptation du génotype au terrain pour cobtenir des arbres de qualité du point de vue de la couleur.

Nous pensons que les forestiers possèdent maintenant avec le prélèvement et le tranchage des carottes de sondage un moyen efficace et objectif de mesurer la couleur $d u$ bois, en forêt, dès l'abattage ou sur le parc à bois en usine.

Or à l'heure actuelle il n'existe aucune mesure de la couleur dans toute la chaîne de fabrication qui mette en cuvre le bois pour son aspect esthétique dans la production de placages, le dessin de projets de meubles ou l'utilisation de panneaux de décoration. Dans une fabrication ou un contrat de vente, seul le nom de l'espèce désigne le meuble ou le produit fini : armoire en chêne, en noyer ou encore parquet de chêne ou de pin, sans qu'il existe aucune quantification des tolérances, des écarts de teintes à respecter pour le produit fabriqué. 
La mesure de la couleur réalisée sur les carottes de sondage prélevées de façon non-destructive sur les arbres offre des avantages aussi bien dans les domaines technologiques, agronomiques et économiques.

\author{
Reçu le 10 septembre 1986. \\ Accepté le 7 octobre 1986.
}

\title{
Remerciements
}

Remerciements à Mademoiselle Voichita Bucur et à la Société Ober-France, Longeville-enBarrois (Meuse).

\author{
Summary \\ Wood colour variability measurement. \\ New method applied on wood increment cores
}

The existing method for measuring the wood colour needs veneers or large solid wood-blocks. The recent development of tester (spectrophotocolorimeter) allows us to do the measurement on smaller samples as well as $9 \mathrm{~mm}$ to $4 \mathrm{~mm}$ width.

The proposed new-method of wood colour measurement concerns the appreciation of the colour of living stand trees, or boles already cut and stored on woodyard, by mean of increment cores of $10 \mathrm{~mm}$ or $5 \mathrm{~mm}$ thickness.

In order to enter upon a comparison study of a large number of trees we propose that the increment cores can be sliced $0.6 \mathrm{~mm}$ thickness using the industrial veneering device.

The new-method consists in placing dry cores in a woodframe grooved in both part in $10 \mathrm{~mm}$ and $5 \mathrm{~mm}$ groove and glued together. The block of glued wood (containing 50 cores even more) is placed in a industrial device to be sliced and to obtain moreover the same veneer surface roughness as veneers from the logs do.

In such a way we obtain, 8 to 10 suitable veneers by each $10 \mathrm{~mm}$ increment core and 2 to 4 for the $5 \mathrm{~mm}$ one, to measure wood colour.

The results show that :

- the measurement is feasible,

- the chromatic characteristics $L, a, b$ calculated from Hunter $L, a, b$ colour system are the same as one would expect for all the veneers cut within the same increment core sample. We did not find significant differences for lightness $L^{*}, F_{36}^{1}=3,10 \mathrm{NS}$ between increment cores of $10 \mathrm{~mm}$ and $5 \mathrm{~mm}$ thick.

The differences existing between trees can be shown for $L^{*}, a^{*}$ and $b^{*}$ as well for the $10 \mathrm{~mm}$ increment cores as for the $5 \mathrm{~mm}$ thick one. The highest interesting characteristic is $\mathrm{L}$ * (lightness) when trees are compared.

Since this new-method has proceeded it would seem that any wood piece of any size and shape can be wood included and cut into veneers and measured for the colour.

Key words: Wood, colour, veneers, variability, spectrophotometry, increment cores. 


\section{Références bibliographiques}

Thiercelin F., Perrin J.R., 1972. Débit d'échantillons d'épaisseur rigoureusement uniforme à l'aide de scies-fraises jumelées. Ann. Sci. For.. 30 (2), 295-299.

JaNin G., 1973. Détermination des diagrammes polaires des rendements et des indices de permanganate sur carottes droites et obliques. Essais de microclassage sur carottes obliques. Document à distribution limitée Station de Recherches sur la Qualité des Bois $\mathbf{n}^{\circ}$ 1973/1974.

Polge H., 1978. Fifteen years of wood radiation densitometry. Wood Science and Technology, 12, 187-196.

Polge H., Thiercelin F., 1979. Growth stress apprais.al through increment core measurements. Wood science, 12 (2), 86-92.

Bucur V., 1983. An ultrasonic method for measuring the elastic constants of wood increment cores bored from living trees. Ultrasonics, May, 116-126.

JANIN G., 1983. Microtests papetiers. Microcuisson, microclassage, microraffinage, mesure automatique de la longueur des fibres. Thèse de Docteur d'Etat ès-Sciences, I.N.P.G., 5 mai.

Perrin J.R., 1983. Dispositif de sciage de carottes de scndage de $5 \mathrm{~mm}$ sans collage préalable sur un support. Ann. Sci. For., $\mathbf{4 0}$ (4), 399-406.

Gentner R., 1985. Appréciation non destructive de la qualité du bois d'arbres sur pied: cas de l'Epicéa de Stika (Picea sitchensis (Bong.) Carr.). Mémoire de $3^{\mathrm{c}}$ année E.N.I.T.E.F., Sept.

JaNiN G., 1985. Etude de la variabilité de la couleur du bois de chêne de tranchage (Quercus sp.). Actes du $5^{\mathrm{c}}$ Congrès de l'Association Internationale de la Couleur. Monte Carlo, 16-22/6/ $1985\left(\mathrm{n}^{\circ} 75\right)$.

Janin G., 1986a. Etude de la variabilité de la couleur du chêne de tranchage. Le Bois National, 11 janvier, 19-21.

Janin G., 1986b. La couleur du bois, un facteur clè de: qualité. Mesures, 17 mars, 61-65.

JANIN G., 1986c. Mesure de la couleur du bois; intérêt agronomique, technologique et économique. Communication présentée à Bâtibois, Bordeaux, 2-3/10/1986.

Hunter R.S., 1975. The measurement of appearance. John Wiley \& Sons, New York.

MosLemi A.A., 1967. Quantitative color measurement for black walnut wood, U.S.F.S. Res. Paper NC-17. North Central Experiment Station Forest Service. U.S.D.A. Saint-Paul, Minnesota, $16 \mathrm{p}$. 